(2) Open Access Full Text Article

\title{
Detection of in situ cleaved pI I5 with the cut specific antibodies in rapid protein inactivation system by tobacco etch viral protease cleavage
}

This article was published in the following Dove Press journal:

Antibody Technology Journal

9 August 20II

Number of times this article has been viewed

\author{
Mayuko Koreishi ${ }^{1,2}$ \\ Yasuko Honjo' \\ Ayano Satoh ${ }^{1,2}$ \\ 'The Research Core for \\ Interdisciplinary Sciences (RCIS), \\ Okayama University, Okayama, Japan \\ ${ }^{2}$ Graduate School of Natural Science \\ and Technology, Okayama University, \\ Okayama, Japan
}

\begin{abstract}
Gene perturbation methods are commonly used in the study of gene and protein function. The authors of this paper recently developed a rapid protein inactivation technique utilizing tobacco etch virus (TEV)-derived protease. TEV protease recognizes the ENLYFQG (Glu-Asn-Leu-Tyr-Phe-Gln-Gly) amino acid sequence and specifically cleaves between Q and G. The authors developed antibodies that recognize the cleaved TEV (ENLYFQ) sequence, both in vitro and in vivo, but do not bind to uncleaved TEV (ENLYFQG). Using these antibodies, in situ protein cleavage was successfully detected. These antibodies used in combination with the TEV protease may be a useful complement to other perturbation methods.
\end{abstract}

Keywords: TEV protease, Golgi, golgins, microinjection, recombinant proteins

\section{Introduction}

The characterization of gene product function is an important challenge in the field of cell biology, especially since the completion of the Human Genome Project. Additionally, research into the causes of human disorders has revealed that defects in intracellular membrane trafficking can cause serious diseases. ${ }^{1,2}$ Therefore, understanding how gene products are transported via membrane trafficking and how trafficking affects their function is crucial.

Ribonucleic acid (RNA) interference (RNAi) is a powerful technique used to disrupt a given messenger RNA using complementary double-stranded RNA, thereby reducing corresponding protein levels. ${ }^{3}$ This technique is applicable for cell-based assays and therefore has been widely used by researchers in the field of cell biology, including membrane trafficking. Although RNAi is useful, there are certain limitations associated with its use. For example, the depletion of the Golgi matrix protein GM130 by RNAi has been described by three different groups and has resulted in contradictory phenotypes. ${ }^{4-6}$

According to published data and our experience, the depletion of Golgi-related proteins by RNAi takes $72-96$ hours, ${ }^{6-8}$ during which time the cells either die or compensate by adjusting the expression levels of other genes. Therefore, any phenotype observed may not reflect the primary loss of function phenotype for the disrupted gene. To address these concerns, we developed a technique for the rapid inactivation of proteins that allows accurate observation of the primary effects of gene product perturbation. ${ }^{9}$

For rapid inactivation, we chose the tobacco etch virus (TEV)-derived protease (TEV protease). TEV protease is unique in its extremely precise cleavage specificity: it recognizes the ENLYFQG (Glu-Asn-Leu-Tyr-Phe-Gln-Gly) consensus sequence and
Correspondence: Ayano Satoh Graduate School of Natural Science and Technology, Okayama University, Okayama, Japan

Tel $+818625 I 8482$

Fax +8I 862518705

Email ayanol13@cc.okayama-u.ac.jp 
cleaves between Q and G. Using nested polymerase chain reaction, a TEV protease recognition site was introduced into a cDNA (complementary deoxyribonucleic acid) expression construct. After transfection, the protein was treated with the TEV protease by microinjection, both in vitro and in situ. Since the TEV recognition sequence is not found in the human gene database, this method can be used to rapidly remove any protein from the cell.

Using this method, we had previously demonstrated that in situ cleavage of $\mathrm{p} 115$, a coat protein I vesicle-tethering protein, caused reduced cargo trafficking. ${ }^{9}$ However, we were unable to demonstrate direct protein cleavage in cells because of a lack of visualization tools. Here we raised anti-TEV (which recognizes only C-terminal ENLYFQ, not ENLYFQG or internal ENLYFQG) antibodies to detect the cleaved TEV sequence and were able to specifically detect in situ protein cleavage. Therefore, the use of these antibodies in combination with TEV protease can be a useful complement to other protein/gene perturbation methods.

\section{Materials and methods}

\section{Antibodies}

The antibodies used in this study are as follows: monoclonal anti-influenza hemagglutinin (anti-HA) (16B12, BAbCO, Richmond, CA), polyclonal anti-giantin, ${ }^{10}$ Alexa-conjugated secondary antibodies (Invitrogen, Carlsbad, CA), and horseradish peroxidase-conjugated secondary antibodies (Pierce, Rockford, IL). Anti-HA agarose was obtained from Sigma (St Louis, MO).

\section{Production of anti-TEV antibodies}

Polyclonal rabbit anti-TEV antibodies were raised using TEV-cleaved hexapeptide (ENLYFQ) as an antigen (Figure 1A). A cysteine residue was added at its N-terminus for conjugation to keyhole limpet hemocyanin (KLH), and 6-aminohexanoic acid (Ahx) was used as a spacer (Figure 1A). Peptide synthesis by Fmoc (fluorenylmethoxycarbonyl) solid phase synthesis, immunization using the KLH-conjugated peptide and Freund's complete adjuvant, and a titer check by enzyme-linked immunosorbent assay (ELISA) were performed by Operon Biotechnologies (Tokyo, Japan). The first and second bleeds were collected a week after the first and second immunizations, respectively. Immediately after the third immunization and bleed, exsanguination was performed. Pooled anti-sera were used in this study.

\section{Plasmids and stable cell lines}

The C-terminal TEV-cleaved sequence (ENLYFQ)-bearing p115 (Figure 1C) was generated by site-directed mutagenesis by introducing a stop codon between $\mathrm{Q}$ and $\mathrm{G}$ of the internal TEV-modified p115 (Figure 1B). The truncated p115 (1-886) and HeLa cells stably expressing the internal TEV-modified p115 were described previously. ${ }^{9}$ We modified green fluorescence protein (GFP)-tagged golgin84 used in the study by Satoh et $\mathrm{al}^{11}$ to insert the TEV cleavage site at the 300th amino acid of golgin-84 (Figure 4A). The internal TEV-modified golgin 84 was expressed transiently by transfection in HeLa cells. ${ }^{9}$ The transient transfection was performed with Lipofectamine ${ }^{\mathrm{TM}}$ LTX (Invitrogen) following the manufacturer's protocol.

\section{Cell culture and microinjection}

Cells were grown in Dulbecco's modified Eagle's medium supplemented with $10 \%$ fetal bovine serum on $12-\mathrm{mm}$ glass coverslips. Desalted TEV protease ( $4 \mathrm{U} / \mu \mathrm{L}$, Invitrogen) together with Alexa-conjugated dextran $(1 \mu \mathrm{g} / \mu \mathrm{L})$ as an injection marker was injected into the cytoplasm. After the microinjection, the cells were incubated for 1.5-3.0 hours at $37^{\circ} \mathrm{C}$ and then processed for immunofluorescence. In typical experiments, 40-100 cells were injected.

\section{Immunoprecipitation (IP) and Western blotting 9,11}

Cell lysates were prepared with IP buffer (10 mM HEPES [4-(2-hydroxyethyl)-1-piperazineethanesulfonic acid]- $\mathrm{KOH}$, $\mathrm{pH} 7.4,100 \mathrm{mM} \mathrm{KCl}, 0.1 \mathrm{mM}$ dithiothreitol [DTT], $2.5 \mathrm{mM}$ $\mathrm{MgCl}_{2}, 1 \%$ Triton $^{\circledR} \mathrm{X}-100$ and the protease inhibitor cocktail from Roche, Penzberg, Upper Bavaria, Germany). After incubation for 10 minutes on ice, the extract was clarified by centrifugation at $14,000 \mathrm{~g}$ for 20 minutes. For IP, the supernatants $(\sim 100 \mu \mathrm{g}$ protein) were incubated with anti-HA agarose (Sigma) or anti-GFP agarose (MBL, Woburn, MA) for 30 minutes at $4^{\circ} \mathrm{C}$. After washing three times with IP buffer, the beads were washed three times with TEV buffer $(50 \mathrm{mM}$ Tris- $\mathrm{HCl}$ [pH 8.0], $0.5 \mathrm{mM}$ ethylenediaminetetraacetic acid, $1 \mathrm{mM}$ DTT). Proteins on the beads were then incubated with/ without TEV protease ( $5 \mathrm{U}$, Invitrogen) for 1 hour at $30^{\circ} \mathrm{C}$. The samples were fractionated by sodium dodecyl sulfate polyacrylamide gel electrophoresis followed by Western blotting, with antibodies indicated in the figures.

\section{Immunofluorescence microscopy, 9}

Cells on coverslips wer e fixed with $3.7 \%$ formalin in phosphate buffered saline (PBS) for 15 minutes and permeabilized with $0.1 \%$ Triton $\mathrm{X}-100$ in PBS for 5 minutes at 


\section{A CAhXENLYFQ}
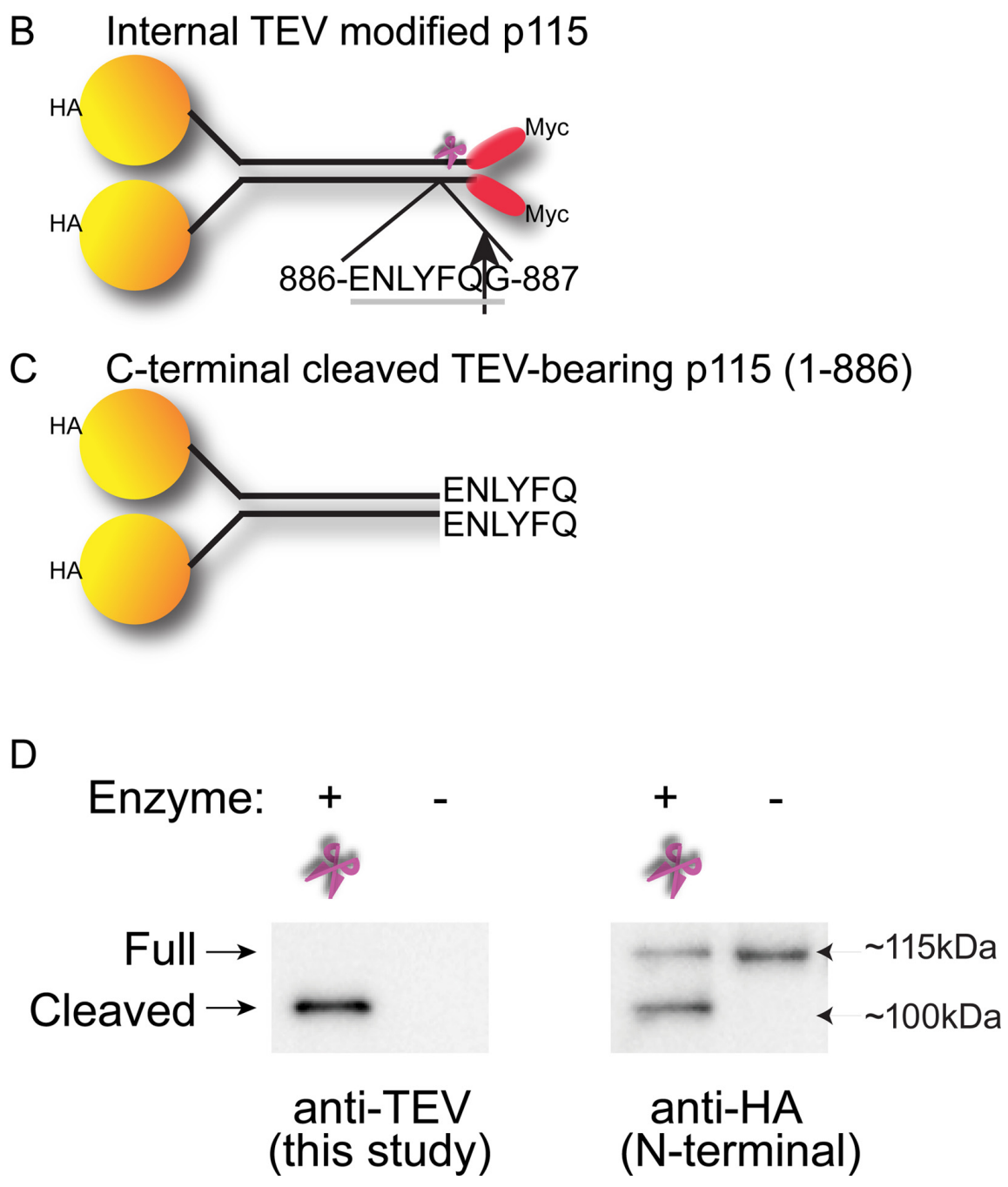

Figure I (A) The antigen peptide. Cysteine was added at the N-terminus for KLH conjugation. Ahx was used as a spacer. (B, C) Schematic models of modified pI I 5 constructs used in this study. The TEV protease recognizes the heptapeptide sequence ENLYFQG and cleaves between $\mathrm{Q}$ and $\mathrm{G}$ with high specificity. This heptapeptide was inserted between amino acids 886 and 887 of bovine pI I 5 (internal TEV-modified p I I5) (B). As a cleaved control, truncated pI I5 (I-886) followed by TEV-cleaved ENLYFQ sequence was constructed (C-terminal-cleaved TEV-bearing pl I 5 (I-886)) (C). Both constructs were tagged with HA at their N-termini for detection. (D) Detection of in-vitro cleavage of internal TEV-modified p I I5. HeLa cells stably expressing the TEV-modified p I I 5 with N-terminal HA tag were used. HA-tagged modified p I 5 was immunoprecipitated with anti-HA-agarose from cell lysates, and immunoreactive complexes were treated with or without the TEV protease for 30 minutes at $30^{\circ} \mathrm{C}$ and analyzed by Western blotting with anti-TEV (left panel) and anti-HA (right panel, to detect the N-termini) antibodies. The anti-TEV antibodies specifically recognized the cleaved protein but not the full-length protein. Abbreviations: Ahx, 6-aminohexanoic acid; ENLYFQG, Glu-Asn-Leu-Tyr-Phe-Gln-Gly; HA, hemagglutinin; KLH, keyhole limpet hemocyanin; TEV, tobacco etch virus.

room temperature. The cells were blocked with $4 \%$ bovine serum albumin (BSA) in PBS for 15 minutes, and then incubated for 15 minutes with primary antibodies diluted in $4 \%$ BSA in PBS. The cells were washed three times with PBS, and incubated for 15 minutes with secondary antibodies conjugated to Alexa Fluor ${ }^{\circledR}$ Dyes (Invitrogen). After washing the cells, the coverslips were mounted on microscope slides and imaged using an FV1000 confocal microscope equipped with a $60 \times$ oil objective (Olympus, Tokyo, Japan).

\section{Results and discussion Production of cleaved TEV (ENLYFQ)-specific antibodies}

In general, sequence epitopes in proteins consist of 6-12 amino acids. ${ }^{12-14}$ Since the cleaved TEV (ENLYFQ) was a hexapeptide, a non-antigenic Ahx was used as a spacer (Figure 1A) to maximize the presentation of the antigen peptide. The titers were determined by ELISA (not shown). The first and second bleeds were collected a week after the first and second 
immunizations, respectively. Immediately after the third immunization and bleed, exsanguination was performed. Pooled anti-sera were used in this study.

\section{Detection of in vitro protein cleavage by anti-TEV antibodies}

To test the specificity of the anti-TEV antibody, in vitro cleavage of the modified p1 15 was performed. The TEV-modified p115 was immunoisolated from HeLa cells stably expressing the modified $\mathrm{p} 115^{9}$ via its N-terminal HA-tag by IP with anti-HA-agarose. Immunoreactive complexes were treated with or without the TEV protease, and analyzed by Western blotting with anti-TEV (Figure 1D, left panel) and anti-HA (Figure 1D, right panel) antibodies. HA staining revealed that the cleavage was incomplete, with approximately $35 \%$ of the modified p115 uncleaved in the condition tested. The antiTEV antibodies specifically recognized the cleaved protein but not full-length p115 (Figure 1D, left panel).
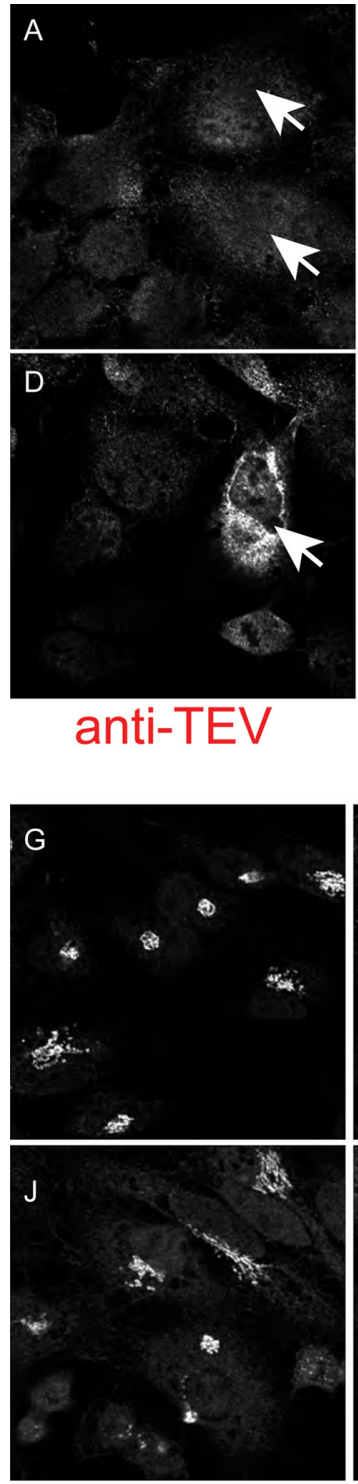

anti-Giantin

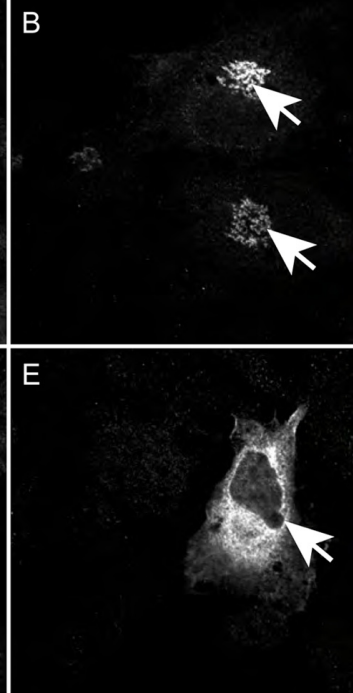

anti-HA
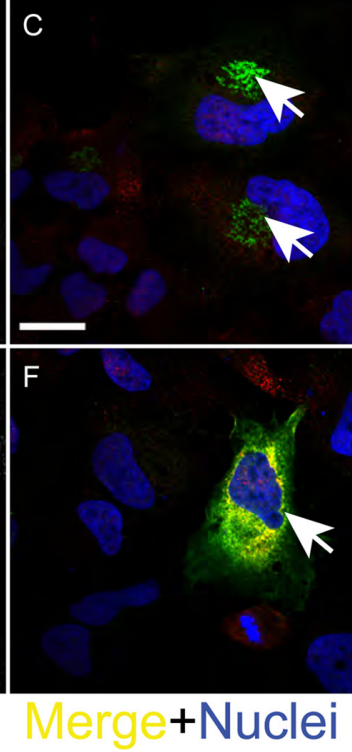

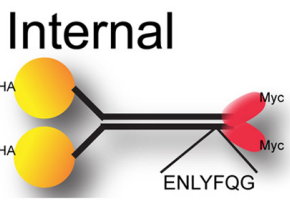

C-term.

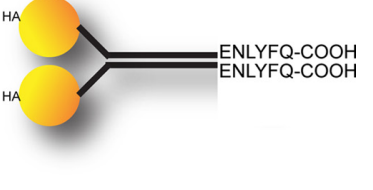

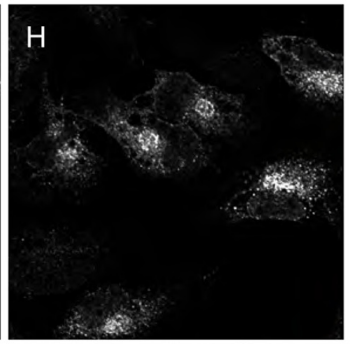
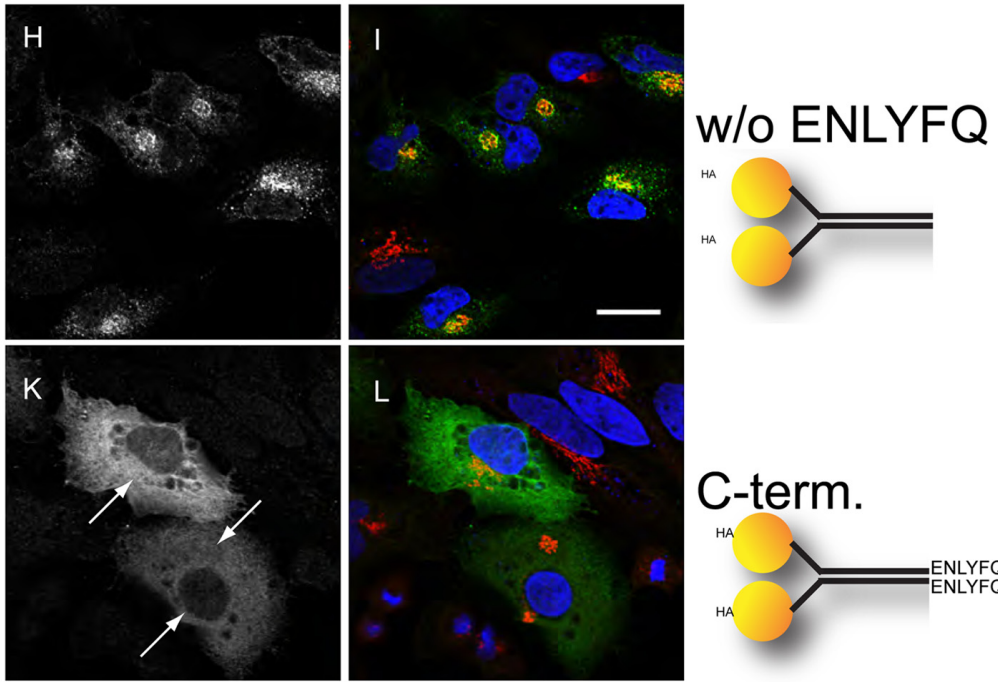

anti-HA

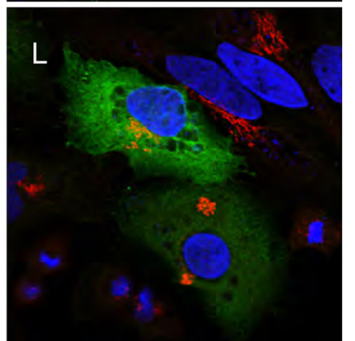

C-term.
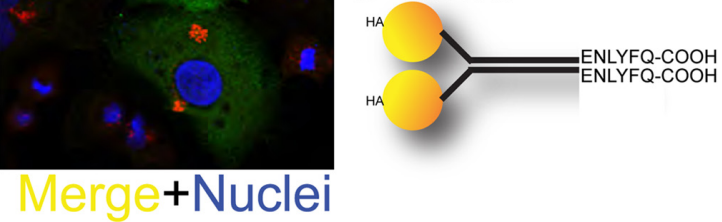

Figure 2 Specificity of anti-TEV antibodies in immunofluorescence applications. HeLa cells transiently expressing the TEV-modified pII5 with N-terminal HA tag (A-C) and C-terminal cleaved TEV-bearing pII5 (D-F) were fixed and immunostained with anti-TEV (left panels, red) and anti-HA (middle panels, green) antibodies. The merged images with nuclear staining (blue) are shown on the right. Cells expressing HA-constructs are indicated with white arrows. The anti-TEV antibodies did not recognize uncleaved proteins in immunofluorescence applications. HeLa cells transiently expressing truncated pII5 (I-886, G-I) and the C-terminal TEVcleaved pII5 (I-886, J-L) were immunostained with anti-giantin (G, J; red, Golgi marker) and anti-HA (H, K; green) antibodies. The merged images with nuclear staining (blue) are shown on the right. The addition of ENLYFQ to the truncated pII5 (I-886) interfered with its localization to the Golgi apparatus (white arrows). Abbreviations: ENLYFQG, Glu-Asn-Leu-Tyr-Phe-Gln-Gly; HA, hemagglutinin; TEV, tobacco etch virus; w/o, without. 


\section{Detection of in situ protein cleavage by anti-TEV antibodies}

To test the specificity of the anti-TEV antibody in immunofluorescence applications, the internal TEV-modified p115 (Figure 1B) and C-terminal TEV-cleaved ENLYFQbearing truncated p115 (1-886) (Figure 1C) were expressed transiently, and cells were immunostained using anti-TEV and anti-HA antibodies. The latter was used to detect N-terminal HA-tags. The anti-TEV antibodies did not recognize the internal TEV-modified p1 15 (Figures 2A-2C), whereas it did recognize the C-terminal TEV-cleaved ENLYFQ-bearing p115 (1-886) (Figures 2D-2F).

Since the authors of this paper noticed that the C-terminal TEV-cleaved ENLYFQ-bearing p115 (1-886) localized throughout the cells, they compared the subcellular localization of this protein to that of p115 (1-886) without ENLYFQ. Both constructs were expressed transiently, and stained for giantin, a Golgi marker (Figures $2 \mathrm{G}$ and $2 \mathrm{~J}$ ), and HA to mark their N-termini (Figures $2 \mathrm{H}$ and $2 \mathrm{~K}$ ). The majority of the truncated p115 (1-886, without ENLYFQ) localized to the Golgi area marked by giantin (Figures $2 \mathrm{G}-2 \mathrm{I}$ ), whereas the C-terminal TEV-cleaved ENLYFQ-bearing p115 did not (Figures 2J-2L). These data suggest that the addition of ENLYFQ may mask the binding site(s) in p115 (1-886) to the Golgi apparatus. These binding sites could be located in the head (1-651 for GBF1, Cog2, and Rab1 bindings), the first coiled-coil (652-701 for SNARE and Rab1 bindings), and/or the second coiled-coil (704-776 for Rab1 binding) domains of $\mathrm{p} 115 .^{15-19}$

Furthermore, in situ protein cleavage was performed by microinjecting the TEV protease into HeLa cells stably expressing the internal TEV-modified p115. To mark the protease-injected cells, Alexa-labeled dextran was co-injected. Two hours after microinjection, cells were fixed and immunostained with anti-TEV and anti-HA antibodies. As shown in Figure 3C, the anti-TEV antibodies marked certain structures and primarily colocalized with HA, suggesting that the anti-TEV antibodies recognized in situ cleaved proteins. Protein cleavage could not be observed in shorter time points ( 20 minutes incubation). This could be because of the lower concentration of anti-TEV antibodies in the antisera used. Therefore, affinity purification of the anti-TEV antibodies may be needed to achieve the high concentrations required for the detection of cleaved proteins at earlier time points. Importantly, microinjection of the TEV protease and dextran did not affect anti-TEV reactivity.

We noticed that in situ cleaved p115 localized to the Golgi within the time observed (Figure 3), unlike C-terminal TEV-cleaved ENLYFQ-bearing p115 (Figure 2). The difference in the localization of these two proteins may be because
A
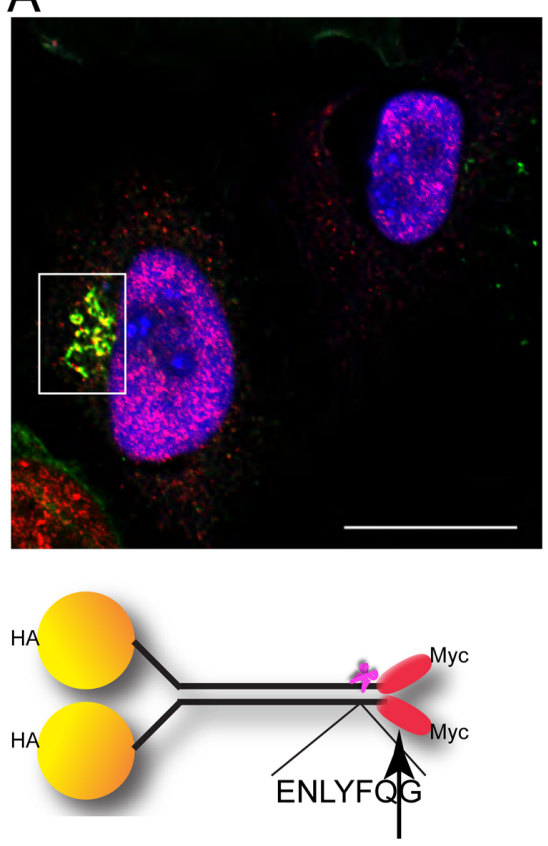

B

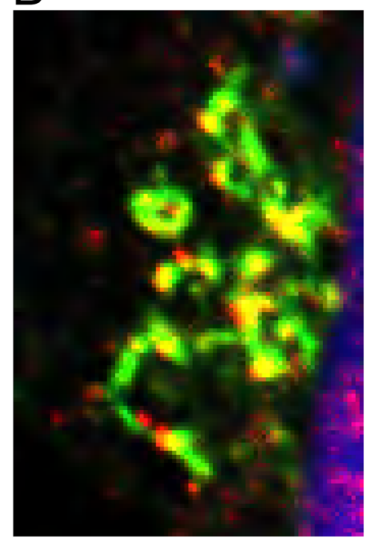

anti-TEV

anti-HA

Injection marker

cleaved protein
C

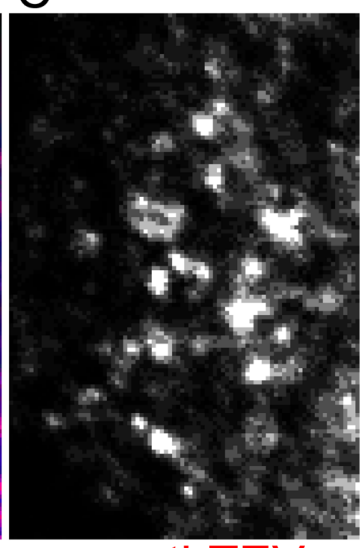

anti-TEV

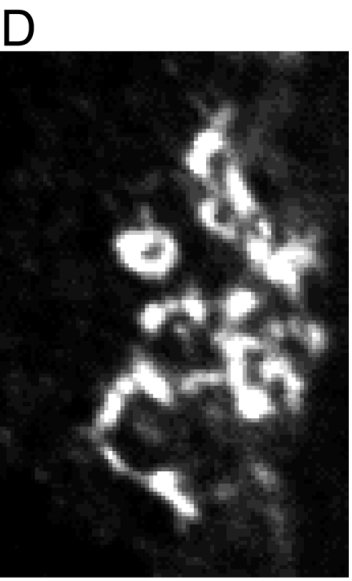

anti-HA

Figure 3 Detection of in-situ protein cleavage by anti-TEV antibodies. HeLa cells stably expressing the internal TEV-modified pI I 5 with N-terminal HA tag were microinjected with the TEV protease and injection marker (blue). After 2 hours incubation, cells were fixed and immunostained with anti-TEV (panel C, red in panels $\mathbf{A}$ and $\mathbf{B}$ ) and anti-HA (panel $\mathbf{D}$, green in panels $\mathbf{A}$ and $\mathbf{B}$ ) antibodies. The merged images are shown in $\mathbf{A}$ and $\mathbf{B}$. Images $\mathbf{B}-\mathbf{D}$ represent a 3.5-fold magnification of the boxed area in A. Bar, $20 \mu \mathrm{m}$. Abbreviations: ENLYFQG, Glu-Asn-Leu-Tyr-Phe-Gln-Gly; HA, hemagglutinin; TEV, tobacco etch virus. 
the in situ cleaved p115 had bound to the Golgi apparatus before the cleavage, and ENLYFQ generated by the cuts may not have interfered with the pre-existing interactions.

Finally, to demonstrate the broad applications of this method, in vitro and in situ protein cleavage were performed using HeLa cells transiently expressing the internal TEV- modified golgin 84, another Golgi-related protein. The TEVmodified golgin 84 was immunoisolated via its $\mathrm{N}$-terminal GFP-tag by IP with anti-GFP-agarose as described above. Immunoreactive complexes were treated with or without the TEV protease, and analyzed by Western blotting with anti-TEV (Figure 4A, left panel) and anti-GFP (Figure 4A,
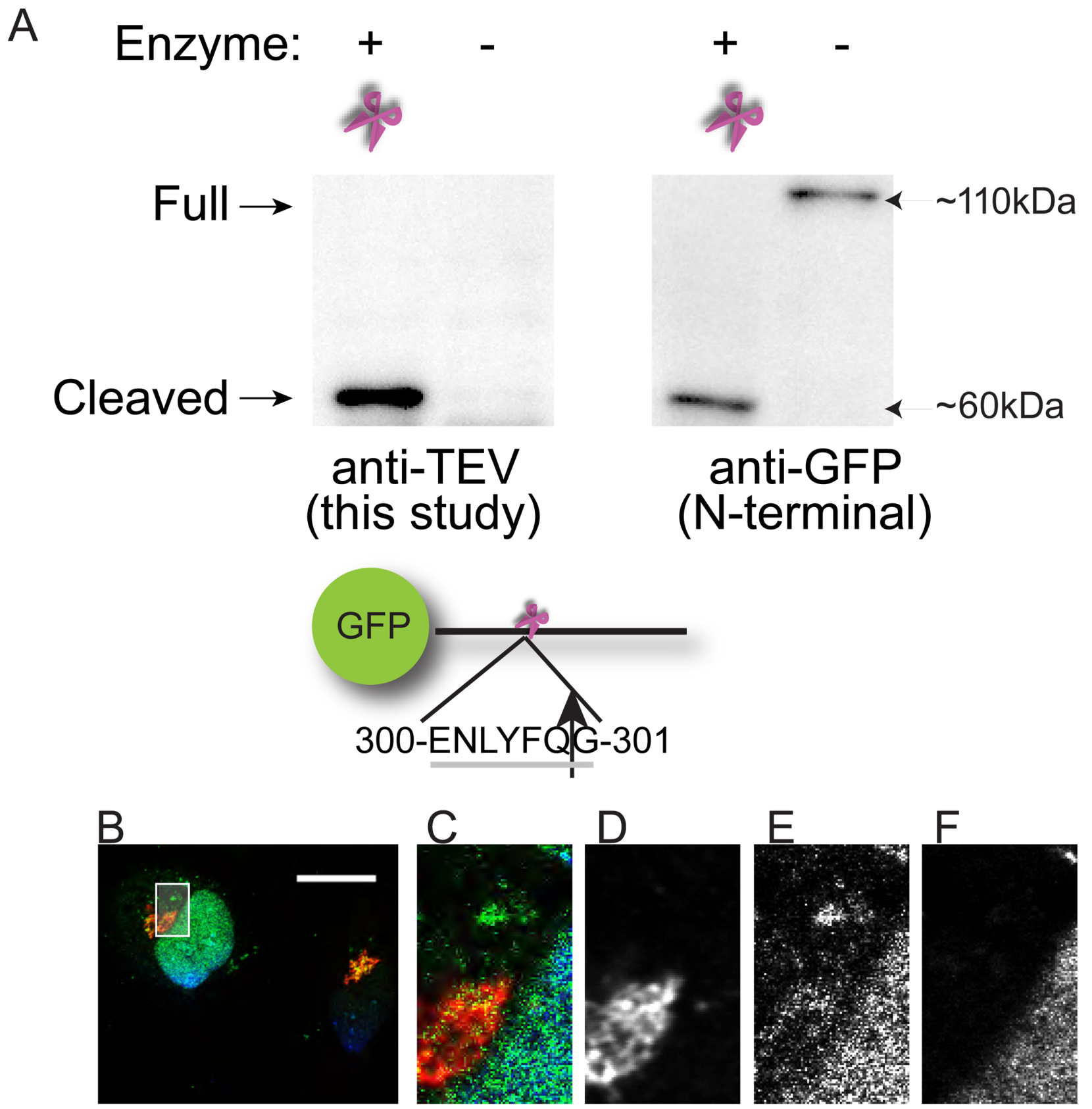

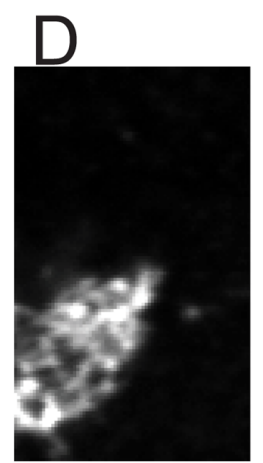

GM130

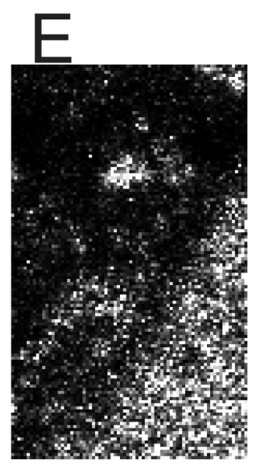

GFP

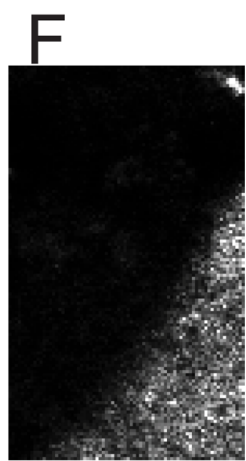

Nucleus

Figure 4 (A) Detection of in vitro cleavage of internal TEV-modified golgin 84. HeLa cells transiently expressing the TEV-modified golgin 84 with N-terminal GFP tag were used. GFP-tagged modified golgin 84 was immunoprecipitated with anti-GFP-agarose from cell lysates, and immunoreactive complexes were treated with or without the TEV protease and analyzed by Western blotting with anti-TEV (left panel) and anti-GFP (right panel, to detect the N-termini) antibodies. The anti-TEV antibodies specifically recognized the cleaved protein but not the full-length protein. (B-F) Detection of in-situ protein cleavage by anti-TEV antibodies. HeLa cells transiently expressing the internal TEV-modified golgin with N-terminal GFP tag were microinjected with the TEV protease and injection marker. After 2 hours incubation, cells were fixed and stained for anti-GMI 30 (panel D, red in panels B and C), anti-TEV (not shown) antibodies, and injection marker (blue, nuclei). Images C-F represent a 5-fold magnification of the boxed area in B. Bar, $20 \mu \mathrm{m}$. Abbreviations: ENLYFQG, Glu-Asn-Leu-Tyr-Phe-Gln-Gly; GFP, green fluorescent protein; TEV, tobacco etch virus. 
right panel) antibodies. The anti-TEV antibodies specifically recognized the cleaved protein but not the full-length golgin (Figure 4A, left panel). Moreover, in situ protein cleavage was performed as described above. As shown in Figures 4B-4F, the majority of GFP signal in the protease-injected cells appeared to localize to the nuclei, and the rest appeared to be very slightly, if any, colocalized with GM130, a Golgi marker. Any specific labeling of anti-TEV antibodies was not observed, suggesting that the in situ cleaved golgin-84 may have defused and been degraded. Golgin-84 has a Golgi localization signal at its C-terminus. ${ }^{20}$ Cleaving the N-terminal part off this domain can cause a change of localization and degradation. For some reason, GFP signals in enzymeinjected cells appeared in their nuclei.

The TEV antibodies could be used for the kinetic analyses of receptor-mediated endocytosis or signaling. This can be achieved by introducing a TEV cleavage site in a receptor of interest, subsequent to which this TEV-receptor construct could be used to transfect cells, allowing for the inactivation of the receptor at the cell surface via the TEV protease cleavage. The inactivated/cleaved receptor could then be detected using fluorescently labeled anti-TEV antibodies. Furthermore, if both the TEV protease and labeled anti-TEV antibodies are added to a cell-culture media, the fluorescence of the surfacebound antibodies could be monitored by live imaging.

\section{Acknowledgments}

We thank the members of the Research Core for Interdisciplinary Sciences for discussion and support, and Risa Matsumoto and Tomoka Kawase for the technical assistance provided. This work was funded by the special Coordination Fund for Promoting Science and Technology of MEXT (Ministry of Education, Culture, Sports, Science and Technology, Japan), Hayashi Memorial Foundation for Female Natural Scientists, Ryobi Teien Memorial Foundation, Naito Foundation, Uehara Memorial Foundation, Kurata Memorial Hitachi Science and Technology Foundation, and Kanae Foundation for the Promotion of Medical Science.

\section{Disclosure}

The authors report no conflicts of interest in this work.

Antibody Technology Journal

\section{Publish your work in this journal}

Antibody Technology Journal is international, peer-reviewed, open access journal publishing original research, reports, reviews and commentaries on all areas of antibody technology. The manuscript management system is completely online and includes a very quick and fair

\section{References}

1. Howell GJ, Holloway ZG, Cobbold C, Monaco AP, Ponnambalam S. Cell biology of membrane trafficking in human disease. Int Rev Cytol. 2006;252:1-69.

2. Mellman I, Nelson WJ. Coordinated protein sorting, targeting and distribution in polarized cells. Nat Rev Mol Cell Biol. 2008;9(11):833-845.

3. Boutros M, Ahringer J. The art and design of genetic screens: RNA interference. Nat Rev Genet. 2008;9(7):554-566.

4. Puthenveedu MA, Bachert C, Puri S, Lanni F, Linstedt AD. GM130 and GRASP65-dependent lateral cisternal fusion allows uniform Golgienzyme distribution. Nat Cell Biol. 2006;8(3):238-248.

5. Marra P, Salvatore L, Mironov A Jr, et al. The biogenesis of the Golgi ribbon: the roles of membrane input from the ER and of GM130. Mol Biol Cell. 2007;18(5):1595-1608.

6. Diao A, Frost L, Morohashi Y, Lowe M. Coordination of golgin tethering and SNARE assembly: GM130 binds syntaxin 5 in a p115-regulated manner. J Biol Chem. 2008;283(11):6957-6967.

7. Diao A, Rahman D, Pappin DJ, Lucocq J, Lowe M. The coiled-coil membrane protein golgin- 84 is a novel rab effector required for Golgi ribbon formation. J Cell Biol. 2003;160(2):201-212.

8. Hicks SW, Horn TA, McCaffery JM, Zuckerman DM, Machamer CE. Golgin-160 promotes cell surface expression of the beta-1 adrenergic receptor. Traffic. 2006;7(12):1666-1677.

9. Satoh A, Warren G. In situ cleavage of the acidic domain from the p115 tether inhibits exocytic transport. Traffic. 2008;9(9):1522-1529.

10. Pelletier L, Jokitalo E, Warren G. The effect of Golgi depletion on exocytic transport. Nat Cell Biol. 2000;2(11):840-846.

11. Satoh A, Wang Y, Malsam J, Beard MB, Warren G. Golgin-84 is a rab1 binding partner involved in Golgi structure. Traffic. 2003; 4(3):153-161.

12. Dyrberg T, Oldstone MB. Peptides as antigens. Importance of orientation. J Exp Med. 1986;164(4):1344-1349.

13. Maelicke A, Plumer-Wilk R, Fels G, et al. Epitope mapping employing antibodies raised against short synthetic peptides: a study of the nicotinic acetylcholine receptor. Biochemistry. 1989;28(3):1396-1405.

14. van Kampen V, Becker WM, Chen Z, et al. Analysis of B-cell epitopes in the N-terminal region of Chi t I component III using monoclonal antibodies. Mol Immunol. 1994;31(15):1133-1140.

15. Garcia-Mata R, Sztul E. The membrane-tethering protein $\mathrm{p} 115$ interacts with GBF1, an ARF guanine-nucleotide-exchange factor. EMBO Rep. 2003;4(3):320-325

16. Sohda M, Misumi Y, Yoshimura S, et al. The interaction of two tethering factors, p1 15 and COG complex, is required for Golgi integrity. Traffic. 2007;8(3):270-284.

17. An Y, Chen CY, Moyer B, et al. Structural and functional analysis of the globular head domain of 115 provides insight into membrane tethering. J Mol Biol. 2009;391(1):26-41.

18. Shorter J, Beard MB, Seemann J, Dirac-Svejstrup AB, Warren G. Sequential tethering of Golgins and catalysis of SNAREpin assembly by the vesicle-tethering protein p115. J Cell Biol. 2002;157(1):45-62.

19. Beard M, Satoh A, Shorter J, Warren G. A cryptic Rab1-binding site in the p115 tethering protein. J Biol Chem. 2005;280(27):25840-25848.

20. Misumi Y, Sohda M, Tashiro A, Sato H, Ikehara Y. An essential cytoplasmic domain for the Golgi localization of coiled-coil proteins with a $\mathrm{COOH}$-terminal membrane anchor. J Biol Chem. 2001;276(9):6867-6873.

\section{Dovepress}

peer-review system. Visit http://www.dovepress.com/testimonials.php to read real quotes from published authors. 